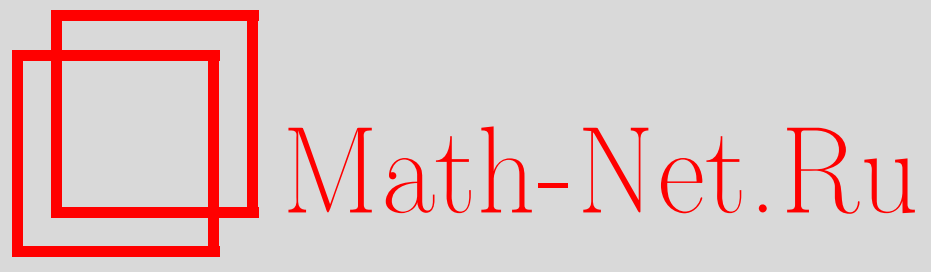

Р. Х. Садикова, Сравнение дискретных смешанных средних, содержащих симметрические функции, Матем. заметки, 2006, том 80, выпуск 2, 262-269

DOI: https://doi.org/10.4213/mzm2807

Использование Общероссийского математического портала Math-Net.Ru подразумевает, что вы прочитали и согласны с пользовательским соглашением http://www . mathnet.ru/rus/agreement

Параметры загрузки:

IP : 34.229 .45 .116

26 апреля 2023 г., 18:03:17

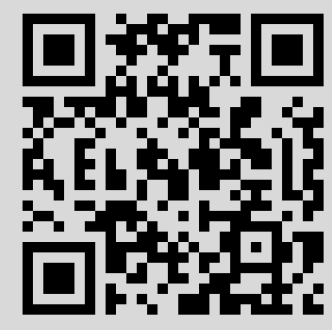




\section{СРАВНЕНИЕ ДИСКРЕТНЫХ СМЕШАННЫХ СРЕДНИХ, СОДЕРЖАЩИХ СИММЕТРИЧЕСКИЕ ФУНКЦИИ}

\section{Р. Х. Садикова}

Для смешанных обобщенных средних доказаны сравнения, из которых выводятся аналоги или обобщения неравенств Несса, Нелсона-Карлсона-Мини, Годуновой, Маркус-Минка и др.

Библиография: 12 названий.

1. Предварительные сведения. В статье для смешанных обобщенных средних доказаны сравнения, из которых выводятся аналоги или обобщения неравенств Несса [1], Нелсона, Карлсона и Мини [2], Годуновой [3], Маркус и Минка [4] и др. Результаты являются продолжением исследований автора.

Пусть $x \in \mathbb{R}_{n}, x=\left(x_{1}, x_{2}, \ldots, x_{n}\right), J_{k}$ - подмножество из $k$ элементов множества $(1,2, \ldots, n)$, мощность $\left|J_{k}\right|=k, f_{1}(\cdot): \mathbb{R} \rightarrow \mathbb{R}$ - строго монотонная непрерывная функция, $f_{1}^{-1}(\cdot)$ - функция, обратная функции $f_{1}(\cdot)$. Обозначим обобщенную среднюю

$$
\mathfrak{M}_{f_{1}}\left(x, J_{k}\right)=f_{1}^{-1}\left(\frac{1}{\left|J_{k}\right|} \sum_{j \in J_{k}} f_{1}\left(x_{j}\right)\right)=f_{1}^{-1}\left(\frac{1}{k} \sum_{j=1}^{k} f_{1}\left(x_{i_{j}}\right)\right)=\mathfrak{M}_{f_{1}}(x, k),
$$

где $i_{1}, i_{2}, \ldots, i_{k} \in J_{k}$.

Если $f_{2}(\cdot): \mathbb{R} \rightarrow \mathbb{R}$ - другая строго монотонная непрерывная функция, а $f_{2}^{-1}(\cdot)$ ей обратная функция, то имеем смешанную обобщенную среднюю вида

$$
\begin{aligned}
\mathfrak{M}_{f_{1}, f_{2}}\left(x, J_{k}\right) & =\mathfrak{M}_{f_{1}, f_{2}}(x, k)=\mathfrak{M}_{f_{2}}\left(\mathfrak{M}_{f_{1}}(x, k)\right) \\
& =f_{2}^{-1}\left(\frac{1}{\left(\begin{array}{c}
n \\
k
\end{array}\right)} \sum_{\left\{J_{k}|| J_{k} \mid=k\right\}} f_{2}\left(f_{1}^{-1}\left(\frac{1}{k} \sum_{j=1}^{k} f_{1}\left(x_{i_{j}}\right)\right)\right)\right),
\end{aligned}
$$

где $\left(\begin{array}{l}n \\ k\end{array}\right)=C_{n}^{k}$ - число сочетаний, т.е. число всех подмножеств по $k$ элементов множества $\left(x_{1}, x_{2}, \ldots, x_{n}\right), k<n$.

В ходе доказательства будут использованы следующие свойства:

(a) $\mathfrak{M}_{f_{1}}\left(\mathfrak{M}_{f_{2}}(x, k)\right) \neq \mathfrak{M}_{f_{2}}\left(\mathfrak{M}_{f_{1}}(x, k)\right)$ при $f_{1}(\cdot) \neq f_{2}(\cdot)$;

(б) $\mathfrak{M}_{f_{1}}\left(\mathfrak{M}_{f_{2}}(x, k)\right) \leqslant \mathfrak{M}_{f_{1}}\left(\mathfrak{M}_{f_{3}}(x, k)\right)$, если $\mathfrak{M}_{f_{2}}(x, k) \leqslant \mathfrak{M}_{f_{3}}(x, k)$, а также ниже приведенное неравенство Иессена [1; с. 90-96].

Теорема Иессена. Пусть две вещественные строго монотонные непрерывные функции $f_{1}(\cdot), f_{2}(\cdot)$ определены на полупрямой $\mathbb{R}_{+}, f_{1}(\cdot)$ переводит $\mathbb{R}_{+}$в $\mathbb{R}_{+}$,

(C) Р. Х. САдиковА, 2006 
функция $f_{2}(\cdot)$ возрастает и выпукла, а также суперпозиция $f_{2} \circ f_{1}^{-1}(\cdot)$ выпукла на $\mathbb{R}_{+}$. Тогда для неотрицателъных чисел $x_{i}, i=1,2, \ldots, n, u p_{i} \geqslant 0, \sum_{i=1}^{n} p_{i}=1$ имеет место неравенство

$$
f_{1}^{-1}\left(\sum_{i=1}^{n} p_{i} f_{1}\left(x_{i}\right)\right) \leqslant f_{2}^{-1}\left(\sum_{i=1}^{n} p_{i} f_{2}\left(x_{i}\right)\right),
$$

т.е. сравнение двух обобщенных средних

$$
\mathfrak{M}_{f_{1}}(x) \leqslant \mathfrak{M}_{f_{2}}(x), \quad x=\left(x_{1}, x_{2}, \ldots, x_{n}\right) .
$$

При $f_{1}(\cdot) \neq f_{2}(\cdot)$ равенство в $(*)$ достигается толъко тогда, когда все $x_{i}, i=$ $1,2, \ldots, n$, равнъ. В случае, если суперпозичия $f_{2} \circ f_{1}^{-1}-$ вогнутая функиия, то неравенство $(*)$ будет противоположным.

Отметим, что при $f_{1}(u)=u, f_{2} \circ f_{1}^{-1}(u)=f_{2}(u)$ - выпуклой функции из неравенства Иессена $(*)$ имеем неравенство Йенсена [5]

$$
f_{2}\left(\sum_{i=1}^{n} p_{i} x_{i}\right) \leqslant \sum_{i=1}^{n} p_{i} f_{2}\left(x_{i}\right)
$$

которое также будет использовано при выводе общих результатов и их частных случаев.

ЗАмечАниЕ. Неравенство Иессена $(*)$ было доказано Шиша и Карго [6] при условии, что выпуклость $f_{2} \circ f_{1}^{-1}(\cdot)$ гарантирует следующее предложение о функциях $f_{1}(\cdot), f_{2}(\cdot)$ : функция

$$
x(\cdot) \rightarrow \frac{f_{2}(x(\cdot))}{f_{1}^{\prime}(x(\cdot))}, \quad f_{1}^{\prime}(x(\cdot)) \neq 0 \quad \forall x(\cdot),
$$

1) не убывает при одинаковой монотонности функций $f_{1}(\cdot), f_{2}(\cdot)$, или

2) не возрастает при различной монотонности функций $f_{1}(\cdot), f_{2}(\cdot)$.

Эти условия успешно применяются при выводе частных случаев из общих результатов.

\section{2. Общие результаты.}

ТеОрема 1. Пусть $x \in \mathbb{R}_{n}, x=\left(x_{1}, x_{2}, \ldots, x_{n}\right), J_{k}$ - подмножество $k$ элементов множества $(1,2, \ldots, n)$, мощность $\left|J_{k}\right|=k, 1<k<n, i_{j} \in J_{k}, j \in 1,2, \ldots, k$, $f_{i}(\cdot), i=1 \div 4,-$ строго монотонные непрерывные функции на $\mathbb{R}_{+}$и при этом

$$
\begin{aligned}
& \mathfrak{M}_{f_{1}}(x, k) \leqslant \mathfrak{M}_{f_{3}}(x, k), \\
& \mathfrak{M}_{f_{2}}(x, k) \leqslant \mathfrak{M}_{f_{4}}(x, k) .
\end{aligned}
$$

Тогда

$$
\mathfrak{M}_{f_{1}, f_{2}}(x, k) \leqslant \mathfrak{M}_{f_{3}, f_{4}}(x, k)
$$

m.e.

$$
\begin{aligned}
& f_{2}^{-1}\left(\frac{1}{\left(\begin{array}{c}
n \\
k
\end{array}\right)} \sum_{\left\{J_{k}|| J_{k} \mid=k\right\}} f_{2}\left(f_{1}^{-1}\left(\frac{1}{k} \sum_{j=1}^{k} f_{1}\left(x_{i_{j}}\right)\right)\right)\right) \\
& \quad \leqslant f_{4}^{-1}\left(\frac{1}{\left(\begin{array}{c}
n \\
k
\end{array}\right)} \sum_{\left\{J_{k}|| J_{k} \mid=k\right\}} f_{4}\left(f_{3}^{-1}\left(\frac{1}{k} \sum_{j=1}^{k} f_{3}\left(x_{i_{j}}\right)\right)\right)\right) .
\end{aligned}
$$


Равенство в (3) достигается только в случае:

1) $x_{1}=x_{2}=\cdots=x_{n}$, либо

2) $n-k+1$ элементов из $\left(x_{1}, x_{2}, \ldots, x_{n}\right)$ равны нулю.

Теорема 2. Пусть $x \in \mathbb{R}_{n}, x=\left(x_{1}, x_{2}, \ldots, x_{n}\right), J_{k}$ - подмножество $k$ элементов множества $(1,2, \ldots, n)$, мощность $\left|J_{k}\right|=k, i_{j} \in J_{k}, j=1,2, \ldots, k$, $\left|J_{k-1}\right|=k-1,2<k<n, i_{j} \in J_{k-1}, j=1,2, \ldots, k-1, f_{i}(\cdot), i=1 \div 4,-$ строго монотонные непрерывные функции на $\mathbb{R}_{+}, f_{4} \circ f_{3}^{-1}(\cdot)$ - выпуклая, $f_{4}(\cdot)$ - возрастаящая функиия и при этом

$$
\begin{aligned}
& \mathfrak{M}_{f_{1}}(x, k) \leqslant \mathfrak{M}_{f_{3}}(x, k), \\
& \mathfrak{M}_{f_{2}}(x, k) \leqslant \mathfrak{M}_{f_{4}}(x, k) .
\end{aligned}
$$

Тогда

$$
\mathfrak{M}_{f_{1}, f_{2}}(x, k) \leqslant \mathfrak{M}_{f_{3}, f_{4}}(x, k-1)
$$

m.e.

$$
\begin{aligned}
& f_{2}^{-1}\left(\frac{1}{\left(\begin{array}{c}
n \\
k
\end{array}\right)} \sum_{\left\{J_{k}|| J_{k} \mid=k\right\}} f_{2}\left(f_{1}^{-1}\left(\frac{1}{k} \sum_{j=1}^{k} f_{1}\left(x_{i_{j}}\right)\right)\right)\right) \\
& \quad \leqslant f_{4}^{-1}\left(\frac{1}{\left(\begin{array}{c}
n \\
k-1
\end{array}\right)} \sum_{\left\{J_{k-1}|| J_{k-1} \mid=k-1\right\}} f_{4}\left(f_{3}^{-1}\left(\frac{1}{k-1} \sum_{j=1}^{k-1} f_{3}\left(x_{i_{j}}\right)\right)\right)\right) .
\end{aligned}
$$

Равенство достигается только в следующих случаях:

1) $x_{1}=x_{2}=\cdots=x_{n}$, либо

2) $n-k+2$ элементов из $\left(x_{1}, x_{2}, \ldots, x_{n}\right)$ равны нулю.

Теорема 3. Пусть $x \in \mathbb{R}_{n}, x=\left(x_{1}, x_{2}, \ldots, x_{n}\right), J_{k}$ - подмножество $k$ элементов множества $(1,2, \ldots, n)$, мощность $\left|J_{k}\right|=k,\left|J_{k-1}\right|=k-1,2<k<n, f_{i}(\cdot)$, $i=1 \div 4$, - строго монотонные непрерывные функции на $\mathbb{R}_{+}, f_{2} \circ f_{1}^{-1}(\cdot)$ - вогнутая, $f_{2}(\cdot)$ - возрастающая функиии и при этом

$$
\begin{aligned}
& \mathfrak{M}_{f_{1}}(x, k) \leqslant \mathfrak{M}_{f_{3}}(x, k), \\
& \mathfrak{M}_{f_{2}}(x, k) \leqslant \mathfrak{M}_{f_{4}}(x, k) .
\end{aligned}
$$

Тогда

$$
\mathfrak{M}_{f_{3}, f_{4}}(x, k) \leqslant \mathfrak{M}_{f_{1}, f_{2}}(x, k-1),
$$

m.e.

$$
\begin{aligned}
& f_{4}^{-1}\left(\frac{1}{\left(\begin{array}{c}
n \\
k
\end{array}\right)} \sum_{\left\{J_{k}|| J_{k} \mid=k\right\}} f_{4}\left(f_{3}^{-1}\left(\frac{1}{k} \sum_{j=1}^{k} f_{3}\left(x_{i_{j}}\right)\right)\right)\right) \\
& \quad \leqslant f_{2}^{-1}\left(\frac{1}{\left(\begin{array}{c}
n \\
k-1
\end{array}\right)} \sum_{\left\{J_{k} \| J_{k-1} \mid=k-1\right\}} f_{2}\left(f_{1}^{-1}\left(\frac{1}{k-1} \sum_{j=1}^{k-1} f_{1}\left(x_{i_{j}}\right)\right)\right)\right) .
\end{aligned}
$$

Равенство достигается только в случаях, когда 
1) $x_{1}=x_{2}=\cdots=x_{n}$, либо

2) $n-k+2$ элементов из $\left(x_{1}, x_{2}, \ldots, x_{n}\right)$ равны нулю.

ДокАЗАТЕЛЬство теоремы 1. Докажем неравенство (3). Последовательно применяя неравенство (1) и свойство (б), а затем неравенство (2), получим

$$
\begin{aligned}
\mathfrak{M}_{f_{1}, f_{2}}(x, k) & \triangleq \mathfrak{M}_{f_{2}}\left(\mathfrak{M}_{f_{1}}(x, k)\right)=f_{2}^{-1}\left(\frac{1}{\left(\begin{array}{c}
n \\
k
\end{array}\right)} \sum_{\left\{J_{k}|| J_{k} \mid=k\right\}} f_{2}\left(f_{1}^{-1}\left(\frac{1}{k} \sum_{j=1}^{k} f_{1}\left(x_{i_{j}}\right)\right)\right)\right) \\
& \leqslant f_{2}^{-1}\left(\frac{1}{\left(\begin{array}{c}
n \\
k
\end{array}\right)} \sum_{\left\{J_{k}|| J_{k} \mid=k\right\}} f_{2}\left(f_{3}^{-1}\left(\frac{1}{k} \sum_{j=1}^{k} f_{3}\left(x_{i_{j}}\right)\right)\right)\right) \\
& \leqslant f_{4}^{-1}\left(\frac{1}{\left(\begin{array}{c}
n \\
k
\end{array}\right)} \sum_{\left\{J_{k} \| J_{k} \mid=k\right\}} f_{4}\left(f_{3}^{-1}\left(\frac{1}{k} \sum_{j=1}^{k} f_{3}\left(x_{i_{j}}\right)\right)\right)\right) \\
& \leqslant \mathfrak{M}_{f_{4}}\left(\mathfrak{M}_{f_{3}}\left(x, J_{k}\right)\right)=\mathfrak{M}_{f_{4}}\left(\mathfrak{M}_{f_{3}}(x, k)\right)=\mathfrak{M}_{f_{3}, f_{4}}(x, k),
\end{aligned}
$$

где $x=\left(x_{1}, x_{2}, \ldots, x_{n}\right), k<n$.

В ходе доказательства были применены неравенства Иессена (1), (2), в которых достигается равенство только при $x_{1}=x_{2}=\cdots=x_{n}$ (см. выше теорему Иессена). Итак, первое условие равенства в неравенстве $(3)$ - это равенство всех $x_{i}$, $i=1,2, \ldots, n$.

Далее, если $n-k+1$ элементов из $\left(x_{1}, x_{2}, \ldots, x_{n}\right)$ равны нулю, то в каждое подмножество из $k$ элементов войдет один нулевой элемент. Поэтому при этом условии в неравенстве (3) достигается равенство.

Теорема 1 доказана.

ДоказАтельство теоремы 2. Докажем неравенство (6). Вначале заметим, что из $k$ различных элементов, выбранных из данных $n$ элементов, можно составить $k$ различных сочетаний по $k-1$ элементов в каждом. Во всех составленных так сочетаниях любой элемент участвует $k-1$ раз (так как он входит в каждое сочетание, кроме того, из которого выброшен). Поэтому справедливо равенство

$$
(k-1) \sum_{j=1}^{k} f\left(x_{i_{j}}\right)=\sum_{j=1}^{k} \sum_{l=1}^{k-1} f\left(x_{i_{j}}^{l}\right) .
$$

Далее последовательно применяя неравенство (4) со свойством (6), неравенство (5), равенство (10) и, наконец, используя неравенство Йенсена (**) для выпуклой суперпозиции $f_{4} \circ f_{3}^{-1}(\cdot)$ при условии, что функция $f_{4}(\cdot)$ возрастающая (а, следовательно, 
и обратная ей функция $f_{4}^{-1}(\cdot)$ возрастающая), получим

$$
\begin{aligned}
\mathfrak{M}_{f_{1}, f_{2}}(x, k) & =\mathfrak{M}_{f_{2}}\left(\mathfrak{M}_{f_{1}}(x, k)\right) \stackrel{(4),(\delta)}{\leqslant} \mathfrak{M}_{f_{2}}\left(\mathfrak{M}_{f_{3}}(x, k)\right) \\
& \leqslant \mathfrak{M}_{f_{4}}\left(\mathfrak{M}_{f_{3}}(x, k)\right)=f_{4}^{-1}\left(\frac{1}{\left(\begin{array}{c}
n \\
k
\end{array}\right)} \sum_{\left\{J_{k} \| J_{k} \mid=k\right\}} f_{4}\left(f_{3}^{-1}\left(\frac{1}{k} \sum_{j=1}^{k} f_{3}\left(x_{i_{j}}\right)\right)\right)\right) \\
& =f_{4}^{-1}\left(\frac{1}{\left(\begin{array}{c}
n \\
k
\end{array}\right)} \sum_{\left\{J_{k} \| J_{k} \mid=k\right\}} f_{4}\left(f_{3}^{-1}\left(\frac{1}{k} \cdot \frac{1}{k-1} \sum_{j=1}^{k} \sum_{l=1}^{k-1} f_{3}\left(x_{i_{j}}^{l}\right)\right)\right)\right) \\
& \leqslant f_{4}^{-1}\left(\frac{1}{\left(\begin{array}{c}
n \\
k
\end{array}\right)} \sum_{\left\{J_{k} \| J_{k} \mid=k\right\}} \frac{1}{k} \sum_{j=1}^{k} f_{4}\left(f_{3}^{-1}\left(\frac{1}{k-1} \sum_{l=1}^{k-1} f_{3}\left(x_{i_{j}}^{l}\right)\right)\right)\right) \\
& =f_{4}^{-1}\left(\frac{1}{k \cdot C_{n}^{k}} \sum_{\left\{J_{k} \| J_{k} \mid=k\right\}} \sum_{j=1}^{k} f_{4}\left(f_{3}^{-1}\left(\frac{1}{k-1} \sum_{l=1}^{k-1} f_{3}\left(x_{i_{j}}^{l}\right)\right)\right)\right) .
\end{aligned}
$$

Заметим, что двойная сумма в последнем выражении содержит $k \cdot C_{n}^{k}$ слагаемых, но среди них есть одинаковые, а число различных слагаемых равно числу сочетаний из $n$ элементов по $k-1$, т.е. $C_{n}^{k-1}$. Каждое из этих слагаемых повторяется столько раз, в скольких сочетаниях из $k$ элементов, взятых из данных $n$ элементов, содержатся соответствующие $k-1$ элементов. Отсюда ясно, что число повторений каждого слагаемого совпадает с числом элементов, остающихся от $n$ элементов после отбора из них $k-1$ элементов, т.е. $n-k+1$. Поэтому последнее выражение примет вид

$$
\begin{aligned}
f_{4}^{-1}( & \left.\frac{n-k+1}{k \cdot C_{n}^{k}} \sum_{\left\{J_{k-1}|| J_{k-1} \mid=k-1\right\}} f_{4}\left(f_{3}^{-1}\left(\frac{1}{k-1} \sum_{l=1}^{k-1} f_{3}\left(x_{i_{j}}^{l}\right)\right)\right)\right) \\
& =f_{4}\left(\frac{1}{C_{n}^{k-1}} \sum_{\left\{J_{k-1}|| J_{k-1} \mid=k-1\right\}} f_{4}\left(f_{3}^{-1}\left(\frac{1}{k-1} \sum_{l=1}^{k-1} f_{3}\left(x_{i_{j}}^{l}\right)\right)\right)\right) \\
& =\mathfrak{M}_{f_{4}}\left(\mathfrak{M}_{f_{3}}\left(x, J_{k-1}\right)\right)=\mathfrak{M}_{f_{3}, f_{4}}(x, k-1) .
\end{aligned}
$$

При доказательстве неравенства (6) были применены неравенства Иессена (4), (5) и неравенство Йенсена $(* *)$ для выпуклой комбинации $f_{4} \circ f_{3}^{-1}(\cdot)$. А они все достигают равенства только при $x_{1}=x_{2}=\cdots=x_{n}$. Это и есть первое условие равенства доказанного неравенства (6). А также, если $n-k+2$ элементов из $\left(x_{1}, x_{2}, \ldots, x_{n}\right)$ равны нулю, то в подмножества по $k-1$ элементов войдет один нулевой элемент. Поэтому равенство в неравенстве (6) достигается и при условии, если $n-k+2$ элементов из множества $\left(x_{1}, x_{2}, \ldots, x_{n}\right)$ равны нулю. Теорема 2 доказана.

ДокАЗАТЕЛЬСтво тЕоремы 3 проводится так же, как и доказательство теоремы 2, с той лишь разницей, что при применении неравенства Йенсена (**) используется вогнутость комбинации $f_{2} \circ f_{1}^{-1}(\cdot)$ при возрастающей функции $f_{2}(\cdot)$ (а следовательно, и обратная функция $f_{2}^{-1}(\cdot)$ возрастающая).

3. Некоторые частные случаи общих результатов. Исследованиями оценок типа общих результатов (3), (6), (9), как было отмечено выше, занимались Несс [1], Годунова [3], Карлсон, Мини, Нельсон [2]. Выделим некоторые неравенства названных авторов из общих результатов. 
СЛЕДСТВИЕ 1 (ТЕОРемЫ 1 ). Пусть $x \in \mathbb{R}_{n},\left(x_{1}, x_{2}, \ldots, x_{n}\right), J_{k}$ - подмножество из $k$ элементов множества $(1,2, \ldots, n)$, мощность $\left|J_{k}\right|=k, i_{j} \in J_{k}, j=1,2, \ldots, k$, $\left(\begin{array}{c}n \\ k\end{array}\right)=C_{n}^{k}$. Тогда имеет место неравенство Несса [1]

$$
\frac{1}{\left(\begin{array}{c}
n \\
k
\end{array}\right)} \sum_{\left\{J_{k}|| J_{k} \mid=k\right\}}\left(\prod_{j=1}^{k} x_{i_{j}}\right)^{1 / k} \leqslant\left(\frac{1}{\left(\begin{array}{c}
n \\
k
\end{array}\right)} \sum_{\left\{J_{k}|| J_{k} \mid=k\right\}} \prod_{j=1}^{k} x_{i_{j}}\right)^{1 / k} .
$$

Равенство достигается только в случаях, когда

1) $x_{1}=x_{2}=\cdots=x_{n}$, либо

2) $n-k+1$ элементов из $\left(x_{1}, x_{2}, \ldots, x_{n}\right)$ равны нулю.

В теореме 1 при $f_{1}(u)=f_{3}(u)=\ln u, f_{2}(u)=u, f_{4}(u)=u^{k}$ условие (1) обращается в равенство, а условие (2) выполняется по неравенству Иессена (*). Отсюда общий результат (3) и будет сформулированным неравенством Несса.

СЛЕДСТВИЕ 2 (Теоремы 1 ). Пусть $x \in \mathbb{R}_{n}, x=\left(x_{1}, x_{2}, \ldots, x_{n}\right), J_{k}$ - подмножество из $k$ элементов множества $(1,2, \ldots, n), 1<k<n$, мощность $\left|J_{k}\right|=k$, $i_{j} \in J_{k}, j=1,2, \ldots, k,\left(\begin{array}{c}n \\ k\end{array}\right)=C_{n}^{k}, \varphi(\cdot)$ - выпуклая функция на $\mathbb{R}_{+}, a \varphi^{-1}(\cdot)$ - ее обратная функиия. Тогда имеет место неравенство Годуновой [3]

$$
\frac{1}{\left(\begin{array}{c}
n \\
k
\end{array}\right)} \sum_{\left\{J_{k}|| J_{k} \mid=k\right\}} \varphi\left(\frac{1}{k} \sum_{j=1}^{k} \varphi^{-1}\left(x_{i_{j}}\right)\right) \leqslant\left(\frac{1}{\left(\begin{array}{c}
n \\
k
\end{array}\right)} \sum_{\left\{J_{k}|| J_{k} \mid=k\right\}}\left(\varphi\left(\frac{1}{k} \sum_{j=1}^{k} \varphi^{-1}\left(x_{i_{j}}\right)\right)\right)^{k}\right)^{1 / k} .
$$

Равенство достигается только при

1) $x_{1}=x_{2}=\cdots=x_{n}$, либо

2) $n-k+1$ элементов из $\left(x_{1}, x_{2}, x_{n}\right)$ равны нулю.

В теореме 1 при $f_{2}(u)=u, f_{4}(u)=u^{k}, k>1, f_{1}^{-1}(u)=f_{3}^{-1}(u)=\varphi(u)$ выпуклой функции, $\left(f_{1}(u)=f_{3}(u)=\varphi^{-1}(u)-\right.$ вогнутая функция) условие (1) обращается в равенство, а условие $(2)$ выполняется по неравенству Иессена $(*)$. Поэтому общий результат (3) и станет неравенством Годуновой.

СЛЕДСТВИЕ 3 (Теоремы 1). Пусть $x \in \mathbb{R}_{n}, x=\left(x_{1}, x_{2}, \ldots, x_{n}\right), J_{k}$ - подмножество из $k$ элементов множества $(1,2, \ldots, n), 1<k<n$, мощность $\left|J_{k}\right|=k$, $i_{j} \in J_{k}, j=1,2, \ldots, k, 0<s \leqslant t, 0<q \leqslant p,\left(\begin{array}{c}n \\ k\end{array}\right)=C_{n}^{k}$. Тогда имеет место неравенство

$$
\left(\frac{1}{\left(\begin{array}{c}
n \\
k
\end{array}\right)} \sum_{\left\{J_{k}|| J_{k} \mid=k\right\}}\left(\frac{1}{k} \sum_{j=1}^{k}\left(x_{i_{j}}\right)^{q}\right)^{s / q}\right)^{1 / s} \leqslant\left(\frac{1}{\left(\begin{array}{c}
n \\
k
\end{array}\right)} \sum_{\left\{J_{k}|| J_{k} \mid=k\right\}}\left(\frac{1}{k} \sum_{j=1}^{k}\left(x_{i_{j}}\right)^{p}\right)^{t / p}\right)^{1 / t} .
$$

Равенство достигается только в случаях, когда

1) $x_{1}=x_{2}=\cdots=x_{n}$, либо

2) $n-k+1$ элементов из $\left(x_{1}, x_{2}, \ldots, x_{n}\right)$ равны нулю.

В теореме 1 при $f_{1}(u)=u^{q}, f_{3}(u)=u^{p}, 0<q \leqslant p, f_{2}(u)=u^{s}, f_{4}(u)=u^{t}$, $0<s \leqslant t$, условия (1) и $(2)$ теоремы выполняются по неравенству Иессена $(*)$. Общий результат (3) обращается в приведенное неравенство. 
СЛЕДСТВИЕ 4 (ТЕОРемы 2). Пусть $x \in \mathbb{R}_{n}, x=\left(x_{1}, x_{2}, \ldots, x_{n}\right), J_{k}-$ подмножество из $k$ элементов множества $(1,2, \ldots, n), 2<k<n$, мощность $\left|J_{k}\right|=k$, $a\left|J_{k-1}\right|=k-1, i_{j} \in J_{k}, j=1,2, \ldots, k, i_{j} \in J_{k-1}, j=1,2, \ldots, k-1,\left(\begin{array}{c}n \\ k\end{array}\right)=C_{n}^{k}-$ число сочетаний из $n$ элементов по $k$ элементов. Тогда имеет место неравенство Карлсона и других [2]:

$$
\frac{1}{\left(\begin{array}{c}
n \\
k
\end{array}\right)} \sum_{\left\{J_{k}|| J_{k} \mid=k\right\}}\left(\prod_{j=1}^{k} x_{i_{j}}\right)^{1 / k} \leqslant \frac{1}{\left(\begin{array}{c}
n \\
k-1
\end{array}\right)} \sum_{\left\{J_{k-1}|| J_{k-1} \mid=k-1\right\}}\left(\prod_{j=1}^{k-1} x_{i_{j}}\right)^{1 /(k-1)} .
$$

Равенство достигается только в случаях, когда

1) $x_{1}=x_{2}=\cdots=x_{n}$, либо

2) $n-k+2$ элементов из $\left(x_{1}, x_{2}, \ldots, x_{n}\right)$ равны нулю.

В теореме 2 при $f_{1}(u)=f_{3}(u)=\ln u\left(f_{1}^{-1}(u)=f_{3}^{-1}(u)=e^{u}\right), f_{2}(u)=f_{4}(u)=$ $u$ неравенства (4) и (5) обращаются в равенство, а $f_{4} \circ f_{3}^{-1}(u)=e^{u}-$ выпуклая и $f_{4}(u)=u$ - возрастающая функции. Тогда общий результат (6) обращается в приведенное неравенство.

СЛЕДСТВИЕ 5 (теоремЫ 2). Пусть $x \in \mathbb{R}_{n}, x=\left(x_{1}, x_{2}, \ldots, x_{n}\right), J_{k}-$ подмножество $k$ элементов множества $(1,2, \ldots, n), 2<k<n$, мощности $\left|J_{k}\right|=k$, $\left|J_{k-1}\right|=k-1, i_{j} \in J_{k}, j=1,2, \ldots, k, i_{j} \in J_{k-1}, j=1,2, \ldots, k-1, i_{j} \in J_{k-1}$, $j=1,2, \ldots, k-1,0<q \leqslant p, 0<s \leqslant t, t>p,\left(\begin{array}{c}n \\ k\end{array}\right)=C_{n}^{k}-$ число сочетаний из $n$ элементов по $k$ элементов. Тогда имеет место неравенство

$$
\begin{aligned}
& \left(\frac{1}{\left(\begin{array}{c}
n \\
k
\end{array}\right)} \sum_{\left\{J_{k} \| J_{k} \mid=k\right\}}\left(\frac{1}{k} \sum_{j=1}^{k} x_{i_{j}}^{q}\right)^{s / q}\right)^{1 / s} \\
& \leqslant\left(\frac{1}{\left(\begin{array}{c}
n \\
k-1
\end{array}\right)} \sum_{\left\{J_{k-1}|| J_{k-1} \mid=k-1\right\}}\left(\frac{1}{k-1} \sum_{j=1}^{k-1} x_{i_{j}}^{p}\right)^{t / p}\right)^{1 / t} .
\end{aligned}
$$

Равенство достигается только в случаях, когда

1) $x_{1}=x_{2}=\cdots=x_{n}$, либо

2) $n-k+2$ элементов из $\left(x_{1}, x_{2}, \ldots, x_{n}\right)$ равны нулю.

При $s=t, p=q, t>p$ из приведенного неравенства имеем неравенство КарлсонаМини-Нельсона [5; с. 348].

В теореме 2 при $f_{1}(u)=u^{q}, f_{3}(u)=u^{p}, 0<q \leqslant p$, и $f_{2}(u)=u^{s}, f_{4}(u)=u^{t}$, $0<s \leqslant t$ условия (4) и (5) выполняются по неравенству Иессена $(*)$, а комбинация $f_{4} \circ f_{3}^{-1}(u)=(u)^{\frac{t}{p}}$ при $t>p$ является выпуклой и $f_{3}(u)=u^{p}, p>0$, возрастающей функциями. Поэтому из общего результата (6) и получим сформулированное неравенство.

СлеДСТВИе 6 (Теоремы 3 ). Пусть $x \in \mathbb{R}_{n}, x=\left(x_{1}, x_{2}, \ldots, x_{n}\right), J_{k}$ - множество из $k$ элементов множества $(1,2, \ldots, n), 2<k<n$, мощности $\left|J_{k}\right|=k$, $\left|J_{k-1}\right|=k-1, i_{j} \in J_{k-1}, j=1,2, \ldots, k-1,\left(\begin{array}{c}n \\ k\end{array}\right)=C_{n}^{k}-$ число сочетаний из $n$ элементов по $k$ элементов.

Тогда имеет место неравенство Карлсона и других [5]:

$$
\left(\prod_{\left\{J_{k}|| J_{k} \mid=k\right\}} \frac{1}{k} \sum_{j=1}^{k} x_{i_{j}}\right)^{1 /\left(\begin{array}{c}
n \\
k
\end{array}\right)} \geqslant\left(\prod_{\left\{J_{k-1}|| J_{k-1} \mid=k-1\right\}} \frac{1}{k-1} \sum_{j=1}^{k-1} x_{i_{j}}\right)^{1 /\left(\begin{array}{c}
n \\
k-1
\end{array}\right)} .
$$


Равенство достигается толъко в случая к, когда

1) $x_{1}=x_{2}=\cdots=x_{n}$, либо

2) $n-k+2$ элементов из $\left(x_{1}, x_{2}, \ldots, x_{n}\right)$ равны нулю.

Это неравенство следует из общего результата (9) теоремы 3, если положить $f_{1}(u)=f_{3}(u)=u, f_{2}(u)=f_{4}(u)=\ln u \quad\left(f_{2}^{-1}(u)=f_{4}^{-1}(u)=e^{u}\right)$. Условия теоремы (7) и (8) обращаются в равенство, комбинация $f_{2} \circ f_{1}^{-1}(u)=\ln u$ - вогнутая и $f_{2}(u)=\ln u$ - возрастающая функции. А общий результат (9) переходит в нужное неравенство.

\section{СПИСОК ЦИТИРОВАННОЙ ЛИТЕРАТУРЫ}

[1] W. Ness, "Das arithemetische und das geometrische Mittel", Prax. Math., 8:11 (1964), 293-296.

[2] B. Carlson, R. Meany, S. Nelson, "Mixed arithmetic and geometric means", Pacif. J. Math., 38:2 (1971), 343-349.

[3] Е. К. Годунова, Неравенства с выпуклыми функииями, Дисс. ... канд. физ.-матем. наук, МГПИ, М., 1965.

[4] М. Маркус, Х. Минк, Обзор теории матрии, и матричных неравенств, Наука, М., 1972.

[5] Г. Харди, Дж. Литтлвуд, Г. Полиа, Неравенства, ИЛ, М., 1948.

[6] O. Shisha, G. Cargo, "On comparable means", Pacif. J. Math., 4:3 (1964), 1053-1059. 